Les ANNALES POLONICI MATHEMATICI constituent une continuation des ANNALES DE LA SOCIETE POLONAISE DE MATHÉMATIQUE (vol. I-XXV) fondées en 1921 par Stanisław Zaremba.

Les ANNALES POLONICI MATHEMATICI publient, en langues des congrès internationaux, des travaux consacrés à l'Analyse Mathématique, la Géométrie et la Théorie des Nombres. Chaque volume paraît en 3 fascicules.

Les manuscrits dactylographiés sont à expédier à l'adresse : Rédaction des ANNALES POLONICI MATHEMATICI KRAK ÓW (Pologne), ul. Solskiego 30.

Toute la correspondance concernant l'échange et l'administration est à expédier à l'adresse:

ANNALES POLONICI MATHEMATICI

WARSZAWA 10 (Pologne), ul. Sniadeckich 8.

Le prix de ce fascicule est $2 \$$.

Les ANNALES sont à obtenir par l'intermédiaire de ARS POLONA

WARSZaWA (Pologne), Krakowskie Przedmieście 7.

PRINTED IN POLAND

\section{Sur la limitation et l'unicité des solutions des problèmes de Fourier pour un système non linéaire d'équations paraboliques.}

\author{
par J. SzARskI (Kraków)
}

Dans une note antérieure [1], nous arons obtenu certaines limitations et quelques critères d'unicité des solutions des problèmes de Fourier pour un système d'équations paraboliques aux dérivées partielles du second ordre de la forme

$$
\begin{aligned}
& \text { (0.1) } \frac{\partial z_{i}}{\partial t}=f_{i}\left(t, x_{1}, \ldots, x_{n}, z_{1}, \ldots, z_{m}, \frac{\partial z_{i}}{\partial x_{1}}, \ldots, \frac{\partial z_{i}}{\partial x_{n}}, \ldots, \frac{\partial^{2} z_{i}}{\partial x_{j} \partial x_{k}}, \ldots\right) \\
& (i=1,2, \ldots, m),
\end{aligned}
$$

où la $i$-ème équation ne contient pas de dérivées des fonctions $z_{1}, \ldots, z_{i-1}$, $z_{i+1}, \ldots, z_{m}$. Les solutions étaient supposées définies dans le produit topologique $R$ d'un intervalle $0<t<T$ et d'un ensemble ouvert et borné $G$ situé dans l'espace $\left(x_{1}, \ldots, x_{n}\right)$.

Dans la présente note nous nous proposons de généraliser les résultats concernant le premier et le troisième problème de Fourier, en remplaçant le produit topologique $R$ par un ensemble $\Omega$ dont la définition sera donnée au paragraphe 1 . Dans le même paragraphe nous démontrons un lemme qui est une modification du lemme 1.1 de la note [1]. Ce lemme sert à la démonstration des théorèmes $2.1,2.2$ et 3.1 qui sont formulés dans les paragraphes 2 et 3 et qui constituent les généralisations des théorèmes $2.1,2.3$ resp. $2.2,2.4$ et $3.1,3.2$ de la note [1].

§ 1. DÉfinition DE L'ENSEMble $\Omega$. Nous considérons un ensemble $\Omega$ dans l'espace $\left(t, x_{1}, \ldots, x_{n}\right)$ jouissant des propriétés suivantes:

a) $\Omega$ est ouvert, situé dans la couche $0<t<T$, où $T \leqslant+\infty$, et pour chaque $t_{0}, 0 \leqslant t_{0}<T$, la portion de $\Omega$ contenue dans la couche $0 \leqslant t \leqslant t_{0}$ est bornée.

b) Pour chaque $t_{0}, 0 \leqslant t_{0}<T$, l'intersection de la fermeture de $\Omega$ avec le plan $t=t_{0}$ est non vide; nous désignons par $S_{t}$ la projection de cette intersection sur le plan $t=0$. 
c) Pour chaque $t_{0}, 0 \leqslant t_{0}<T$, et pour tout point $X_{0} \in S_{t_{0}}$ on peut faire correspondre à toute suite $t_{v}, v=1,2, \ldots$, telle que $0<t_{v}<T$ et $t_{v} \rightarrow t_{0}$, une suite de points $X_{v}$ de façon que $X_{v} \in S_{t_{v}}$ et $X_{v} \rightarrow X_{0}$.

DÉfINITION DE L'ENSEMbete $\Sigma$ ET $\Sigma_{\alpha}$. Nous désignons par $\Sigma$ la partie de la frontière de $\Omega$ que est située dans la couche $0<t<T$.

Etant donnée une fonction $\alpha\left(t, x_{1}, \ldots, x_{n}\right)$ définie sur $\Sigma$ nous désignons par $\Sigma_{\alpha}$ le sous-ensemble de $\Sigma$ (qui peut être vide ou identique à $\Sigma$ ) dans lequel on a $\alpha>0$.

LEMTE 1.1. Soit $\varphi(t, X)$, où $X=\left(x_{1}, \ldots, x_{n}\right)$, une fonction continue dans la fermeture de l'ensemble $\Omega$ qui vient d'être défini. Posons pour $0 \leqslant t<T$

$$
M(t)=\max _{X_{\theta} S_{t i}} \varphi(t, X) .
$$

Nous disons que:

I. La fonction $M(t)$ est continue dans l'intervalle $0 \leqslant t<T$.

II. Si $M(t)=\varphi(t, X)$, où le point $(t, X)$ appartient à l'intérieur de $\Omega$, et si la dérivée partielle $\varphi_{t}(t, X)$ existe, on a l'inégalité

$$
\bar{D}_{-} M(t) \leqslant \varphi_{t}(t, X),
$$

où $\bar{D}_{-}$désigne le nombre dérivé supérieur à gauche.

Démonstration. I. Soit $0 \leqslant t_{0}<T$ et $t_{\nu}, v=1,2, \ldots$, une suite quelconque telle que $0<t_{v}<T, t_{v} \rightarrow t_{0}$. Il suffit de montrer qu'il existe une suite partielle $t_{v_{\mu}}, \mu=1,2, \ldots$, telle que

$$
M\left(t_{\boldsymbol{v}_{\mu}}\right) \rightarrow M\left(t_{0}\right) .
$$

D'après a) et b) (cf. la définition de l'ensemble $\Omega$ ) et en vertu de la continuité de $\varphi(t, X)$, il existe un point $\bar{X}_{v} \in S_{t_{v}}$ tel que

$$
M\left(t_{v}\right)=\varphi\left(t_{v}, \bar{X}_{v}\right)
$$

et, d'une façon analogue, un point $X_{0} \in S_{t_{0}}$ tel que

$$
M\left(t_{0}\right)=\varphi\left(t_{0}, X_{0}\right) \text {. }
$$

En vertu de a) nous pouvons extraire une suite partielle $\bar{X}_{v_{\mu}}$ de telle façon que

$$
\bar{X}_{v_{\mu}} \rightarrow \bar{X}_{0} \epsilon S_{t_{0}} .
$$

On a alors, vu la continuité de $\varphi(t, X)$,

$$
\varphi\left(t_{v_{\mu}}, \bar{X}_{v_{\mu}}\right) \rightarrow \varphi\left(t_{0}, \bar{X}_{0}\right) \text {. }
$$

Pour achever la démonstration de (1.1), il suffit de prouver que

$$
\varphi\left(t_{0}, \bar{X}_{0}\right)=M\left(t_{0}\right) \text {. }
$$

Or, en vertu de c), il existe une suite de points $X_{v_{\mu}} \in S_{t_{v_{\mu}}}$ telle que

et par conséquent

$$
X_{v_{\mu}} \rightarrow X_{0}
$$

$$
\varphi\left(t_{v_{\mu}}, X_{v_{\mu}}\right) \rightarrow \varphi\left(t_{0}, X_{0}\right)=M\left(t_{0}\right) .
$$

D'autre part, nous arons

$$
\varphi\left(t_{v_{\mu}}, X_{v_{\mu}}\right) \leqslant M\left(t_{v_{\mu}}\right)=\varphi\left(t_{v_{\mu}}, \bar{X}_{v_{\mu}}\right)
$$

Les relations (1.2), (1.4) et (1.5) impliquent 1'inégalité

$$
\varphi\left(t_{0}, \bar{X}_{0}\right) \geqslant M\left(t_{0}\right)
$$

d'où l'on obtient (1.3).

La démonstration de la partie II de notre lemme est tout à fait analogue à celle du lemme 1.1 de la note [1].

\section{§ 2. THÉORÈmE 2.1. Supposons que}

$1^{0}$ Les fonctions $f_{i}\left(t, X, z_{1}, \ldots, z_{m}, p_{1}, \ldots, p_{n}, r_{11}, r_{12}, \ldots, r_{n n}\right), g_{i}(t$, $\left.X, z_{1}, \ldots, z_{m}, p_{1}, \ldots, p_{n}, r_{11}, r_{12}, \ldots, r_{n n}\right)(i=1,2, \ldots, m)$ sont définies dans un ensemble $D$ dont la projection sur le plan $\left(t, x_{1}, \ldots, x_{n}\right)$ recouvre l'ensemble $\Omega$ défini au paragraphe 1 .

$2^{\circ}$ Lorsque les valeurs $\bar{r}_{j k}$ et $\overline{\bar{r}}_{j k}$ sont telles que

$$
\sum_{j, k=1}^{n}\left(\bar{r}_{j k}-\overline{\bar{r}}_{j k}\right) \lambda_{j} \lambda_{k} \leqslant 0 \quad \text { pour tout système } \lambda_{1}, \ldots, \lambda_{n},
$$

alors on a les inégalités

$$
\begin{aligned}
& f_{i}\left(t, X, z_{1}, \ldots, z_{m}, p_{1}, \ldots, p_{n}, \ldots, \bar{r}_{j k}, \ldots\right)- \\
& \quad-f_{i}\left(t, X, z_{1}, \ldots, z_{m}, p_{1}, \ldots, p_{n}, \ldots, \overline{\bar{r}}_{j k}, \ldots\right) \leqslant 0,
\end{aligned}
$$

et des inégalités analogues pour les fonctions $g_{i}$.

$$
3^{\circ} \quad \mid f_{i}\left(t, X, u_{1}, \ldots, u_{m}, p_{1}, \ldots, p_{n}, \ldots, r_{j k}, \ldots\right)-
$$

$-g_{i}\left(t, X, v_{1}, \ldots, v_{m}, p_{1}, \ldots, p_{n}, \ldots, r_{j k}, \ldots\right) \mid \leqslant \sigma_{i}\left(t,\left|u_{1}-v_{1}\right|, \ldots,\left|u_{m}-v_{m}\right|\right)$, où les fonctions $\sigma_{i}\left(t, y_{1}, \ldots, y_{m}\right)$ sont continues et non négatives pour $0 \leqslant t<T$, $y_{i} \geqslant 0(i=1,2, \ldots, m)$ et la fonction $\sigma_{i}$ est non décroissante par rapport $\grave{a}$ chacune des variables $y_{1}, \ldots, y_{i-1}, y_{i+1}, \ldots, y_{m}$; pour $\varepsilon_{i} \geqslant 0$ nous désignons par

$$
y_{i}=\omega_{i}\left(t, \varepsilon_{1}, \ldots, \varepsilon_{m}\right) \quad(i=1,2, \ldots, m)
$$

l'intégrale supérieure à droite du système d'équations différentielles ordinaires

$$
d y_{i} \mid d t=\sigma_{i}\left(t, y_{1}, \ldots, y_{m}\right) \quad(i=1,2, \ldots, m),
$$


issue du point $\left(0, \varepsilon_{1}, \ldots, \varepsilon_{m}\right)$ et nous supposons que cette intégrale existe dans l'intervalle $0 \leqslant t<T$.

$4^{\circ}$ Les fonctions $u_{i}(t, X)$ et $v_{i}(t, X)(i=1,2, \ldots, m)$ sont continues dans la fermeture de $\Omega$, de classe $C^{1}$ dans $\Omega$ et possèdent des dérivées partielles du second ordre par rapport aux variables $x_{1}, \ldots, x_{n}$ continues dans $\Omega$.

$5^{\circ}$ On a dans $\Omega$

$$
\begin{aligned}
\frac{\partial u_{i}}{\partial t} & =f_{i}\left(t, X, u_{1}, \ldots, u_{m}, \frac{\partial u_{i}}{\partial x_{1}}, \ldots, \frac{\partial u_{i}}{\partial x_{n}}, \ldots, \frac{\partial^{2} u_{i}}{\partial x_{j} \partial x_{k}}, \ldots\right), \\
\frac{\partial v_{i}}{\partial t} & =g_{i}\left(t, X, v_{1}, \ldots, v_{m}, \frac{\partial v_{i}}{\partial x_{1}}, \ldots, \frac{\partial v_{i}}{\partial x_{n}}, \ldots, \frac{\partial^{2} v_{i}}{\partial x_{j} \partial x_{k}}, \ldots\right) \cdot(
\end{aligned}
$$

$6^{0}\left|u_{i}(0, X)-v_{i}(0, X)\right| \leqslant \varepsilon_{i} \quad$ pour $\quad X \in S_{0}$.

$7^{\circ}$ Les fonctions $\alpha_{i}(t, X)$ et $\beta_{i}(t, X)(i=1,2, \ldots, m)$ sont définies sur $\Sigma$ et satisfont aux inégalités

$$
\alpha_{i} \geqslant 0, \quad \beta_{i} \geqslant B_{i}=\text { const }>0 ;
$$

$\grave{a}$ chaque point appartenant $\grave{a} \Sigma_{a}$ correspond une demi-droite $l$ issue de ce point, orthogonale d̀ l'axe $t$ et dont un segment est contenu dans la fermeture de $\Omega$.

$8^{\circ}$ Les fonctions $u_{i}$ et $v_{i}$ admettent une dérivée suivant la demi-droite $l$ en tout point de l'ensemble $\Sigma_{\alpha}$ et vérifient les inégalités

$$
\left|\alpha_{i}(t, X) \partial\left[u_{i}(t, X)-v_{i}(t, X)\right] / \partial l-\beta_{i}(t, X)\left[u_{i}(t, X)-v_{i}(t, X)\right]\right| \leqslant \varepsilon_{i} B_{i}
$$
pour $(t, X) \in \Sigma$, où l'on a posé

$$
\partial\left(u_{i}-v_{i}\right) / \partial l \stackrel{\mathrm{d} f}{=} 0 \quad \text { pour } \quad(t, X) \in \Sigma-\Sigma_{a} .
$$

Dans toutes ces hypothèses, les inégalités

$$
\left|u_{i}(t, X)-v_{i}(t, X)\right| \leqslant \omega_{i}\left(t, \varepsilon_{1}, \ldots, \varepsilon_{m}\right) \quad(i=1,2, \ldots, m)
$$

ont lieu dans $\Omega$.

Démonstration. La démonstration est tout à fait analogue à celle du théorème 2.1 resp. 2.3 de la note [1], lorsqu'on s'appuie sur le lemme 1.1 de la présente note. Il n'y a qu'un point de la démonstration qu'il sera utile de reproduire ici. Posons notamment

$$
W_{i}(t)=\max _{X_{6} S_{t}}\left|u_{i}(t, X)-v_{i}(t, X)\right|
$$

(la continuité de $W_{i}(t)$ dans l'intervalle $0 \leqslant t<T$ résulte de la partie I du lemme 1.1) et supposons que pour un indice $i_{0}$ et un $t_{0}, 0<t_{0}<T$, on ait l'inégalité

$$
W_{i_{0}}\left(t_{0}\right)>\omega_{i_{0}}\left(t_{0}, \varepsilon_{1}, \ldots, \varepsilon_{m}\right)
$$

et par exemple

$$
W_{i_{0}}\left(t_{0}\right)=u_{i_{0}}\left(t_{0}, X_{0}\right)-v_{i_{0}}\left(t_{0}, X_{0}\right),
$$

où $X_{0} \in S_{t_{0}}$. Il s'agit de prouver que dans nos hypothèses le point $\left(t_{0}, X_{0}\right)$ est un point intérieur de $\Omega$. Admettons donc, par l'impossible, que $\left(t_{0}, X_{0}\right) \in \Sigma$. D'après $8^{\circ}$ on a alors

(2.4) $\quad \beta_{i_{0}}\left(t_{0}, X_{0}\right)\left[u_{i_{0}}\left(t_{0}, X_{0}\right)-v_{i_{0}}\left(t_{0}, X_{0}\right)\right]$

$$
\leqslant \varepsilon_{i_{0}} B_{i_{0}}+\alpha_{i_{0}}\left(t_{0}, X_{0}\right)\left[\partial\left(u_{i_{0}}-v_{i_{0}}\right) / \partial l\right]_{\left(t_{0}, X_{0}\right)} \text {. }
$$

$D$ 'autre part, d’après (2.2) et la définition de $W_{i}(t)$, on a

$$
\left[\partial\left(u_{i_{0}}-v_{i_{0}}\right) / \partial l\right]_{\left(t_{0}, x_{0}\right)} \leqslant 0,
$$

d'où, selon $7^{\circ}$, on obtient de (2.4) l'inégalité

$$
u_{i_{0}}\left(t_{0}, X_{0}\right)-v_{i_{0}}\left(t_{0}, X_{0}\right) \leqslant \varepsilon_{i_{0}},
$$

ce qui est impossible, puisque en vertu de (2.3) on a

$$
\varepsilon_{i_{0}}<W_{i_{0}}\left(t_{0}\right)=u_{i_{0}}\left(t_{0}, X_{0}\right)-v_{i_{0}}\left(t_{0}, X_{0}\right)
$$

Une conséquence immédiate $d u$ théorème 2.1 est le critère suivant d'unicité de la solution du problème mixte pour le système d'équiations (0.1).

THस́OR亡̀Me 2.2. Maintenons les hypothèses $1^{\circ}, 2^{\circ}, 3^{\circ}$ et $7^{\circ}$, du théorème 2.1 en admettant que

a) $f_{i} \equiv g_{i}$,

b) l'intégrale unique du système (2.1), issue de l'origine, est identiquement nulle, c'est-à-dire

$$
\omega_{\boldsymbol{i}}(t, 0, \ldots, 0) \equiv 0 \quad(i=1,2, \ldots, m) .
$$

Dans ces hypothèses, le problème mixte

$$
\begin{gathered}
z_{i}(0, X)=\varphi_{i}(X) \quad \text { pour } \quad X \in S_{0}, \\
\alpha_{i}(t, X) \frac{\partial z_{i}(t, X)}{\partial l}-\beta_{i}(t, X) z_{i}(t, X)=\psi_{i}(t, X) \quad \text { pour } \quad(t, X) \epsilon \Sigma
\end{gathered}
$$

relatif au système d'équations (0.1), admet dans $\Omega$ au plus une solution continue dans la fermeture de $\Omega$, possédant une dérivée suivant $l$ en tout point de $\Sigma_{a}$ et toutes les dérivées figurant dans (0.1) continues dans $\Omega$. Cette solution (lorsqu'elle existe) dépend d'une façon continue des valeurs initiales $\varphi_{i}(X)$ et des valeurs aux limites $\psi_{i}(t, X)$.

Remarque. Le problème mixte (2.5) devient, en particulier, le premier problème de Fourier lorsque $\alpha_{i} \equiv 0$. Dans le cas où $\alpha_{i} \equiv 1$ et 
la demi-droite $l$ est normale à l'intersection de $\Sigma$ avec le plan dans lequel $l$ est située, le problème (2.5) devient le troisième problème de Fourier.

\$3. Nous allons maintenant formuler un critère d'unicité plus général que celui qui est contenu dans le théorème 2.2.

THÉORÈve 3.1. Supposons que les seconds membres du système d'éinégalités quations (0.1) satisfassent aux hypothèses $1^{\circ}$ et $2^{\circ}$ du théorème 2.1 et aux

$$
\begin{aligned}
& \mid f_{i}\left(t, X, u_{1}, \ldots, u_{m}, p_{1}, \ldots, p_{n}, \ldots, r_{j k}, \ldots\right)- \\
& \quad-f_{i}\left(t, X, v_{1}, \ldots, v_{m}, p_{1}, \ldots, p_{n}, \ldots, r_{j k}, \ldots\right) \mid \leqslant \sigma\left(t, \max _{j}\left|u_{j}-v_{j}\right|\right),
\end{aligned}
$$

où la fonction $\sigma(t, y)$ est continue et non négative pour $0<t<T, y \geqslant 0\left(^{1}\right)$, et admettons que pour chaque intervalle

$$
0<t \leqslant t_{0}
$$

où $0<t_{0}<T$, l'intégrale unique $y(t)$ de l'équation

$$
d y / d t=\sigma(t, y)
$$

définie dans (3.1) et satisfaisant d̀ la condition

$$
\lim _{t \rightarrow 0} y(t)=0,
$$

soit identiquement nulle. Maintenons enfin l'hypothèse $7^{\circ}$ du théorème 2.1 .

Ceci étant admis, le problème mixte (2.5) relatif au système d'équations (0.1) admet dans $\Omega$ au plus une solution continue dans la fermeture de $\Omega$ possédant une dérivée suivant $l$ en tout point de $\Sigma_{\alpha}$ et toutes les dérivées figurant dans (0.1) continues dans $\Omega$.

La démonstration de ce théorème est tout à fait analogue à celle du théorème 3.1 de la note [1]. Il suffit d'y introduire des modifications analogues à celles dont nous avons parlé dans la démonstration du théorème 2.1 de la présente note.

\section{Travaux cités}

[1] J. Szarski, Sur la limitation et l'unicité des solutions d'un système non-linéaire d'équations paraboliques aux dérivées partielles du second ordre, Ann. Polon. Math. 2 (1955), p. 237-249.

\section{INSTYTUT MATEMATYCZNY POLSKIEJ AKADEMIT NAUK}

INSTITUT MATHFMATIQUE DE L'ACADFMTE POLONATSK DFS SOTHNOTS

$$
\text { Regu par la Rédaction le 3. 9. } 1957
$$

\section{Application of the Nörlund summability to the theory of localization for single and double trigonometric series (I)}

\author{
by L. JEŚMA NOWICZ (Toruń)
}

The theory of localization for single trigonometric series developed by Zygmund [1] and that for double trigonometric series developed by Gosselin [2] are restricted to the Cesàro summability. The purpose of this paper is to extend their results by involving a special class of the Nörlund means, containing the Cesàro means as a particular case.

\section{On the Nörlund summability}

Let $\left\{B_{n}\right\}$ be a sequence with non-zero terms for $n$ large enough. We shall say that the series $\sum_{\nu=0}^{\infty} a_{p}$, or the sequence $s_{n}$ of its partial sums, is summable by the Nörlund method $\mathrm{N}\left(B_{n}\right)$, or $\mathrm{N}\left(B_{n}\right)$ summable to the sum $s$, if the following sequence (determined for $n$ large enough)

$$
t_{n}=\frac{\sum_{\nu=0}^{n} B_{n-\nu} a_{\nu}}{B_{n}}=\frac{\sum_{\nu=0}^{n} \Delta B_{n-\nu} s_{\nu}}{B_{n}},
$$

where $\Delta B_{0}=B_{0}, \Delta B_{v}=B_{v}-B_{v-1}$ for $v \geqslant 1$, converges to $s$ as $n \rightarrow \infty$.

In numerous applications we deal with $B_{n}$ positive and non-decreasing for $n$ large enough. For such a sequence the condition

$$
\lim _{n \rightarrow \infty} \frac{B_{n-1}}{B_{n}}=1
$$

is sufficient and necessary for the convergence of the series to involve its $N\left(B_{n}\right)$ summability to the same sum (the condition of regularity).

Let $\left\{A_{n}\right\}$ be a sequence satisfying the following conditions:

1) $A_{n}>0$ for $n \geqslant n_{0}$,

(') La fonction $\sigma(t, y)$ n'est pas supposée continue pour $t=0$.

$$
\begin{gathered}
A_{n} \geqslant A_{n+1} \quad \text { for } n \geqslant n_{0}, \\
\sum_{n=0}^{\infty} A_{n}=\infty
\end{gathered}
$$

